\title{
Laser puncture of symptomatic primary iris pigment epithelial cyst causing hemeralopia: a rare case
}

\author{
Ram Lal Sharma, Mohan Lal Pandey, Vinod Sharma, Kalpana \\ Sharma, Neha Chowdhary \\ Indira Gandhi Medical College \& Hospital, Shimla, Himachal Pradesh, India
}

\begin{abstract}
Primary iris pigment epithelium cysts involve posterior surface of the iris and can occur at pupillary margin or anterior ciliary body. They may be stationary or progressive and sometimes regress spontaneously. These cyst can occasionally lead to angle-closure glaucoma, plateau iris syndrome and secondary pigment dispersion syndrome. A steadily growing cyst may disturb the vision by covering the visual axis and provoke an increase in intraocular pressure (IOP) or even inflammation if it touches the corneal endothelium. There are multiple management options in complicated situations. We report a case of a 17-year-old girl with unusual complaints of hemeralopia and dark-brown discolouration of pupils in both eyes. On examination, she had multiple pigment epithelial iris cysts at the pupillary margins, which were punctured with Nd:Yag laser, and the response was improvement in vision and hemeralopia without any pigment dispersion or IOP changes.
\end{abstract}

Keywords: Nd:Yag laser, primary iris pigment epithelium

\section{Introduction}

Primary iris cyst involves a portion of iris, which is lined by pigment epithelium, while secondary iris cyst are results of some pathology like trauma, inflammations, metastasis, parasites or miotic therapy. Primary iris cysts are less common and mostly congenital, which could be stromal or pigment epithelial cysts. They are of neuroepithelial origin and involve the iris pigment epithelium (IPE) and the ciliary body. Central cysts are rare and account for approximately $3 \%$ of all primary IPE cysts. ' We report a case of bilateral, central pigment epithelial cysts of the iris causing hemeralopia, and cosmetic concern to the patient.

\section{Case}

We report a case of a 17-year-old girl presented with complaints of difficulty in seeing in bright sunlight and brownish discolouration of pupils of both eyes. On slit lamp examination, there were seven visible dark-brown iris cysts with smooth

Correspondence: Dr. Ram Lal Sharma, Professor, Department of Ophthalmology, IGMC, Shimla, Himachal Pradesh, India.

E-mail: rls10@rediffmail.com 

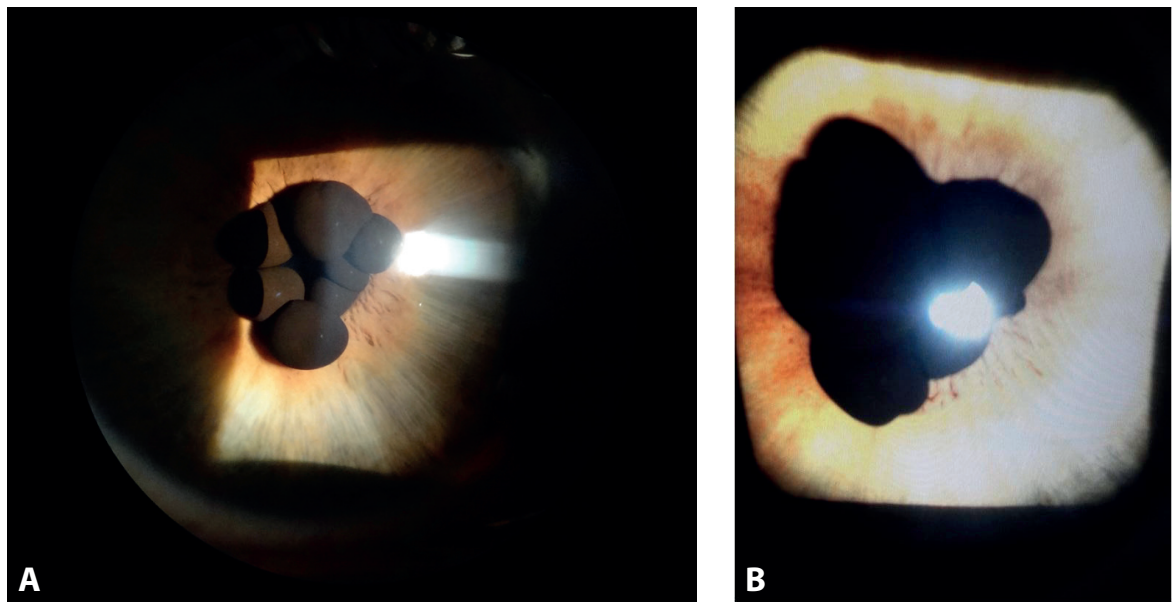

Fig. 1. (A and B): Iris pigment epithelial cysts of right and left eyes.

surface of varying size ranging from 1 to $3 \mathrm{~mm}$ in right eye and six cysts in left eye. They were attached to the pupillary margin and obstructing the pupil so that effective pupil size was just $1 \mathrm{~mm}$ in mesopic state (Fig. $1 \mathrm{~A}$ and $\mathrm{B}$ ). The pupil was round, readily reacting to light and accommodation. During miosis, the lesions often obstructed the pupillary aperture. There were no signs of inflammation in the anterior chamber.

Examination with a Goldmann goniolens showed normal and open angular structures in both eyes. The fundus oculi were normal. Central corneal thickness was $507 \mu \mathrm{m}$ in the right eye and $514 \mu \mathrm{m}$ in the left eye. Intraocular pressure (IOP) was $10.7 \mathrm{mmHg}$ in the right eye and $10.2 \mathrm{mmHg}$ in the left eye. The visual acuity was $6 / 6$ in both eyes without glasses. Anterior segment optical coherence tomography (AS-OCT) showed multiple cystic swellings at the pupillary margin with no peripheral cyst causing angle obstruction (Figs. 2 and 3). The patient was planned for Nd:Yag laser under topical proparacaine $0.5 \%$ in two sittings, 1 week apart, to avoid any reactive inflammation with energy of 0.5 to $1.2 \mathrm{~mJ}$ and two to three shots per cyst to rupture it. The cyst collapsed immediately as fluid oozed out, and the membrane wall of the cyst crumbled towards pupil margin increasing the effective size of the pupil (Fig. 4). There was no pain during the procedures. Post-laser, she was given topical loteprednol four times a day and timolol $(0.5 \%)$ eye drops for a week. There was no recurrence of cyst till 5 months follow up and patient was asymptomatic even in bright light. 


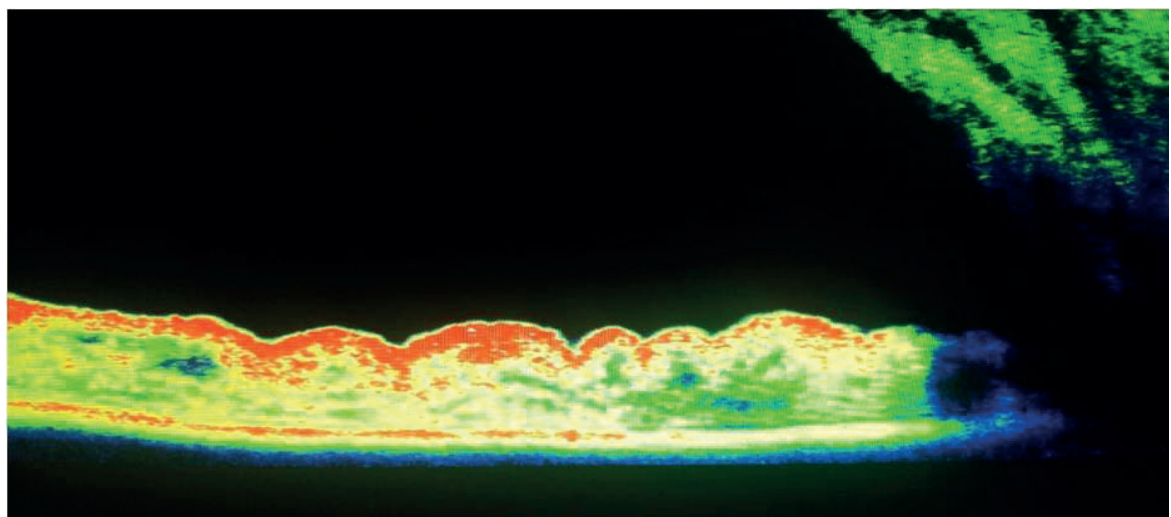

Fig. 2. AS-OCT showing iris pigment epithelial cysts at pupillary margin.

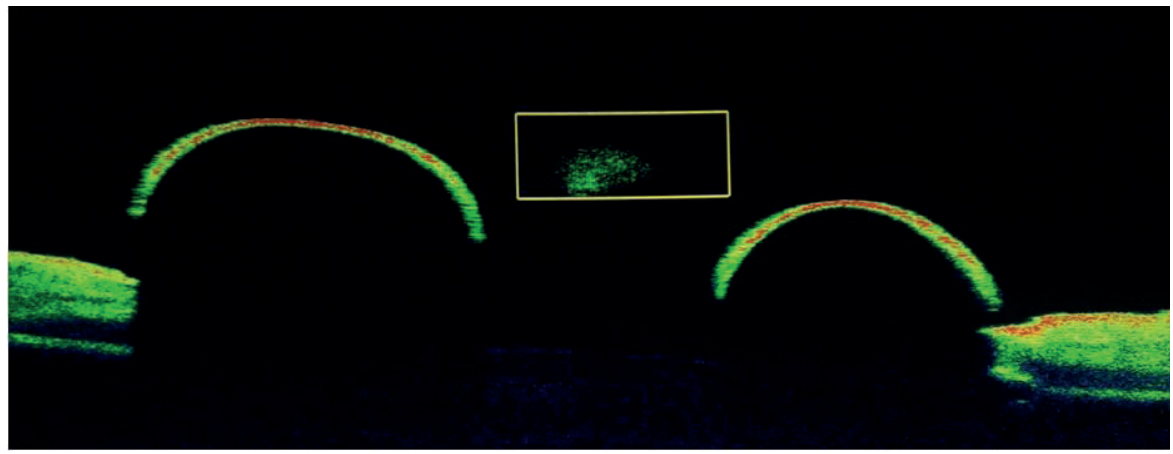

Fig. 3. AS-OCT showing no peripheral cyst causing angle obstruction.

\section{Discussion}

Primary iris cysts are found along the posterior surface of the iris, from the pupillary margin to the anterior ciliary body. Shields distinguishes four types, according to their position in relation to the iris: central, at the pupillary margin; midzonal, between the pupillary margin and the iris root; peripheral, at the iridociliary sulcus, and dislodged, in the anterior chamber or the vitreous cavity. The vast majority of them (76\%) are peripheral. Most of them are stationary and small, and sometimes regress spontaneously. They are usually dark brown, round or oval with a thin wall and sonolucent contents. ${ }^{1}$ The high reflectivity of their wall is attributed to the epithelial cells, while their sonolucent core is compatible with a fluid content.

The origin of primary central IPE cysts is not clear. Their appearance in early childhood in a few familial cases suggests that they may be hereditary with an 


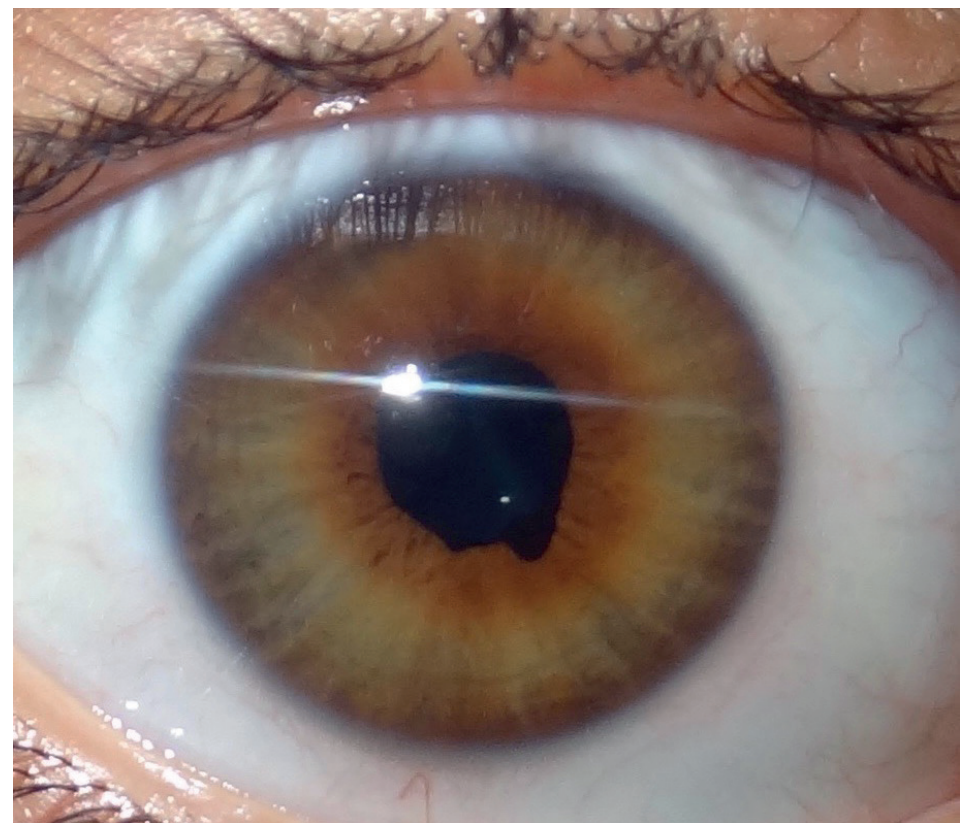

Fig. 4. Post-Nd:Yag laser rupture of three collapsed cysts resulting in clearing of the central visual axis.

autosomal dominant pattern., ${ }^{2,3}$ Lewis et al. described an association with familial aortic dissection. ${ }^{4}$ The proposed theory of formation of these cysts is traction of zonules on the anterior part of optic cup on ciliary epithelium at the fourth month of development. Histologically they are lined by pigmented epithelium and contain clear fluid. This patient neither had any associated systemic diseases nor did other members of her family have similar feature. Primary IPE cysts have often been misdiagnosed as iris or ciliary body melanoma. ${ }^{5}$ Cysts of the iris are more likely to be confused with melanoma than those of ciliary body because of their pigmentation and more anterior location. It was believed that most primary IPE cysts are ophthalmic curiosities that need prolonged observation and no treatment. ${ }^{1}$

The natural course of primary epithelial cysts differs from that of secondary iris cysts, which follow surgical or non-surgical trauma. The latter lesions frequently enlarge and lead to severe complications such as inflammation and glaucoma. A steadily growing cyst, however, may disturb vision by covering the visual axis and provoke an increase in IOP or even inflammation if it touches the corneal endothelium. Their presence is occasionally responsible for plateau iris syndrome and secondary pigment dispersion syndrome. ${ }^{2,3}$ Steps must then be taken to prevent 
or treat complications, such as pupillary obstruction, secondary glaucoma, iridocyclitis, corneal decompensation and loss of vision. These cysts can be ruptured non-surgically by Nd:Yag laser as was done in this case, so that they do not interfere with vision or cause some visual-threatening situations in future.

Different treatment modalities have been suggested for management of these cysts. These include mitomycin $C$ injection into the cyst and needle aspiration with endodiathermy, Nd:Yag laser cystotomy, excision of cysts and intracystic ethanol irrigation. ${ }^{6-10}$ Risks of all treatment options include bleeding, endophthalmitis, cataract formation and cyst recurrence. Perhaps the most concerning risk in treating an epithelial inclusion cyst is the possibility of spilling epithelial cells outside the cyst, which could result in epithelial downgrowth.

We treated this case with $\mathrm{Nd}$ :Yag laser and the response was dramatic. The patient no longer complained of decreased field of vision in bright sunlight. No pigment dispersion or increase in IOP was seen 2 weeks post-laser. There is least possibility of recurrence of these cyst following Yag laser cystotomy, although multiple punctures are unlikely to close and pigment epithelial cyst remains stationary in size when untreated. But no recurrence was observed till 5 months follow up and patient was asymptomatic even in bright light. Thus, Nd:Yag laser is a successful modality for improvement of hemeralopia, providing cosmetic restoration and preventing further complications.

\section{References}

1. Lois N, Shields CL, Shields JA, Mercado G. Primary cysts of the iris pigment epithelium. Ophthalmology. 1998;105:1879-1885.

2. Vela A, Rieser JC, Campbell DG. The heredity and treatment of angle-closure glaucoma secondary to iris and ciliary body cysts. Ophthalmology. 1984;91:332-337.

3. Alward WL, Ossoinig KC. Pigment dispersion secondary to cysts of the iris pigment epithelium. Arch Ophthalmol Chic I. 1995;113:1574-1575.

4. Lewis RA, Merin LM. Iris flocculi and familial aortic dissection. Arch Ophthalmol Chic III. 1995;113:1330-1331.

5. Yanoff M, Zimmerman LE. Pseudo melanoma of anterior chamber caused by implantation of iris pigment epithelium. Arch Ophthalmic Chic III. 1965;74:302-305.

6. Kawaguchi K, Yamamoto S, Nagae Y, Okada AA, Iwasaki N, Tano Y. Treatment of recurrent giant iris cyst with intracyst administration of mitomycin C. Br J Ophthalmol. 2000;84:800-801.

7. Tsai JC, Arrindell EL, O'Day DM. Needle aspiration and endodiathermy treatment of epithelial inclusion cyst of the iris. Am J Ophthalmol. 2001;131:263-265.

8. Kuchenbecker J, Motschmann M, Schmitz K, Behrens-Baumann W. Laser iridocystotomy for bilateral acute angle-closure glaucoma secondary to iris cysts. Am J Ophthalmol. 2000;129:391-393.

9. Verma L, Venkatesh P, Sen S, Lakshmaiah NC, Tewari HK. Surgical removal of a free floating cyst of the iris pigment epithelium causing disturbing visual symptoms. Ophthalmic Surg Lasers. 1999;30:223-225.

10. Behrouzi Z, Khodadoust A. Epithelial iris cyst treatment with intracystic ethanol irrigation. Ophthalmology. 2003;110:1601-1605. 